\title{
An implicit compact finite difference method for the fractional reaction-subdiffusion equation
}

\author{
Behnam Sepehrian *, Mahboobeh Jabbari \\ Department of Mathematics, Faculty of Science, Arak University, Arak 38156-8-8349, Iran \\ *Corresponding author E-mail: b-sepehrian@araku.ac.ir
}

Copyright (C2014 Sepehrian and Jabbari. This is an open access article distributed under the Creative Commons Attribution License, which permits unrestricted use, distribution, and reproduction in any medium, provided the original work is properly cited.

\begin{abstract}
In this article, a high order implicit compact difference method for the fractional reaction-subdiffusion equation is presented. The difference scheme is unconditionally stable and the truncation error is of first order in time and forth order in space. A numerical example is included to demonstrate the validity of theoretical results and efficiency of the scheme.
\end{abstract}

Keywords: Compact finite difference scheme, Fractional reaction-subdiffusion equation, Solvability, Stability, Fourier analysis.

\section{Introduction}

Fractional partial differential equations (FPDEs) have found very applications in various fields of science, for example, physics, chemistry, biology and economics. A fractional reaction-subdiffusion equation (FR-subDE) can be derived from a continuous time random walk model when the transport is dispersive [15] or a continuous time random walk model with temporal memory and sources [5].

In this paper, we consider the following FR-subDE [2]

$\frac{\partial u(x, t)}{\partial t}={ }_{0} D_{t}^{1-\gamma}\left[\kappa_{\gamma} \frac{\partial^{2} u(x, t)}{\partial x^{2}}-\kappa u(x, t)\right]+f(x, t), \quad 0<t \leq T, \quad 0<x<L$,

subject to the boundary and initial conditions

$u(0, t)=\varphi(t), \quad 0<t \leq T$

$u(L, t)=\psi(t), \quad 0<t \leq T$

$u(x, 0)=\phi(x), \quad 0 \leq x \leq L$,

where $\kappa>0$ and $\kappa_{\gamma}>0$ denote constant coefficients and $0<\gamma<1$. Also $\varphi(t), \psi(t)$ and $\phi(x)$ are known functions. ${ }_{0} D_{t}^{1-\gamma} v(0<\gamma<1)$ denotes the Riemann-Liouville fractional derivative of order $1-\gamma$ of the function $v(x, t)$ defined by $[16]$,

${ }_{0} D_{t}^{1-\gamma} v=\frac{1}{\Gamma(\gamma)} \frac{\partial}{\partial t} \int_{0}^{t} \frac{v(x, s)}{(t-s)^{1-\gamma}} d s$. 
Several approximate and numerical solutions for FPDEs are known. Liu et al. [8,9] applied the Method of Lines for solving space FPDEs. In [16] an explicit finite difference method for fractional subdiffusion equation is introduced and a new Van neumann stability analysis is given. Also an implicit numerical scheme for this problem is proposed in [6]. In $[10,12,14]$, some numerical methods for fractional advection-dispersion equation are presented. Chen et al. [2], presented an implicit and an explicit finite differences methods for the problem (1)-(4). However, the truncation errors of their schemes are of first order in time and second order in space.

Compact finite difference (CFD) schemes give us a forth order accuracy to approximate the second order derivatives and they keep the desirable tridiagonal nature of the finite difference equations. Liao et al. [7] implemented a CFD scheme for reaction-diffusion problems. A CFD scheme for the generalized one dimensional Sine-Gordon equation with error analysis was introduced in [4]. A fully implicit CFD method for the fractional diffusion equation with a Fourier analysis was presented in [3].

In this article, an implicit CFD scheme for the problem (1)-(4) is presented and a Fourier analysis is given. We approximate the second-order derivative with respect to space by the CFD, then we use the Grunwald-Letnikov discretization for the approximation of the time fractional derivative. The truncation error of the scheme is of first order in time and fourth order in space.

The paper is organized as follows: In Section 2, the solution of (1)-(4) by implicit CFD is considered. Section 3 is devoted to the matrix form for the difference scheme and the solvability for the linear system of equations. In Section 4, by a Fourier analysis we prove that the scheme is unconditionally stable for all $\gamma$ in the range $0<\gamma<1$. Finally, some numerical results are provided in Section 5. The accuracy and efficiency of the scheme is demonstrated through numerical experiments.

In this paper, we use the "empty sum" convention $\sum_{l=p}^{q} v^{l}=0$ for $q<p$.

\section{2. implicit CFD method for FR-subDE}

Let $\tau=T / N$ be the time step, $t_{k}=k \tau, k=0, \ldots, N, h=L / M$ be the grid step in space and $x_{j}=j h, j=0, \ldots, M$. The familiar central difference quotient

$\frac{1}{h^{2}} \delta_{x}^{2} u_{j}=\frac{1}{h^{2}}\left(u_{j-1}-2 u_{j}+u_{j+1}\right)=\left(\frac{\partial^{2} u}{\partial x^{2}}\right)_{j}+\frac{1}{12}\left(\frac{\partial^{4} u}{\partial x^{4}}\right)_{j} h^{2}+\mathcal{O}\left(h^{4}\right)$,

gives only a second-order approximation to $\frac{\partial^{2} u}{\partial x^{2}}$. By following formula [1]

$$
\begin{aligned}
\left(\frac{\partial^{2} u}{\partial x^{2}}\right)_{j} & =\left[2 h \sinh ^{-1} \frac{\delta_{x}}{2}\right]^{2} u_{j}=\frac{1}{h^{2}}\left[\delta_{x}-\frac{1^{2}}{2^{2} \cdot 3 !} \delta_{x}^{3}+\frac{1^{2} \cdot 3^{2}}{2^{4} \cdot 5 !} \delta_{x}^{5}-\frac{1^{2} \cdot 3^{2} \cdot 5^{2}}{2^{6} \cdot 7 !} \delta_{x}^{7}+\ldots\right]^{2} u_{j} \\
& =\frac{1}{h^{2}}\left(\delta_{x}^{2}-\frac{1}{12} \delta_{x}^{4}+\frac{1}{90} \delta_{x}^{6}-\frac{1}{560} \delta_{x}^{8}+\ldots\right) u_{j},
\end{aligned}
$$

in which $\delta_{x} u_{j}=u_{j+1 / 2}-u_{j-1 / 2}$, and by Eq. (6), we get

$$
\begin{aligned}
\frac{\delta_{x}^{2}}{h^{2}\left(1+\frac{1}{12} \delta_{x}^{2}\right)} u_{j}^{k} & =\frac{1}{h^{2}}\left(\delta_{x}^{2}-\frac{1}{12} \delta_{x}^{4}+\frac{1}{144} \delta_{x}^{6}-\frac{1}{1728} \delta_{x}^{8}+\ldots\right) u_{j}^{k}=\left.\frac{\partial^{2} u}{\partial x^{2}}\right|_{j} ^{k}-\frac{1}{240 h^{2}} \delta_{x}^{6} u_{j}^{k}+\mathcal{O}\left(h^{6}\right) \\
& =\left.\frac{\partial^{2} u}{\partial x^{2}}\right|_{j} ^{k}-\left.\frac{1}{240} \frac{\partial^{6} u}{\partial x^{6}}\right|_{j} ^{k} h^{4}+\mathcal{O}\left(h^{6}\right) .
\end{aligned}
$$

Using the above equation a fourth-order approximation for $\frac{\partial^{2} u}{\partial x^{2}}$ is obtained. For the Riemann-Liouville fractional derivatives, we have [13],

${ }_{0} D_{t}^{1-\gamma} f(t)=\frac{1}{\tau^{1-\gamma}} \sum_{k=0}^{[t / \tau]} \omega_{k}^{(1-\gamma)} f(t-k \tau)+\mathcal{O}\left(\tau^{p}\right)$,

where $\omega_{k}^{(1-\gamma)}$ are the coefficients of the generating function, that is, $\omega(z, \alpha)=\sum_{k=0}^{\infty} \omega_{k}^{(\alpha)} z^{k}$. For $\omega(z, \alpha)=(1-z)^{\alpha}$, these coefficients are $\omega_{0}^{(\alpha)}=1$ and $\omega_{k}^{(\alpha)}=(-1)^{k}\left(\begin{array}{c}\alpha \\ k\end{array}\right)$ and can be evaluated by following recursive formula [11,16],

$\omega_{0}^{(\alpha)}=1, \quad \omega_{k}^{(\alpha)}=\left(1-\frac{\alpha+1}{k}\right) \omega_{k-1}^{(\alpha)}, \quad k \geq 1$. 
Also, in this case $p=1$ in (8). For the convenience of notations, we define $\lambda_{l} \equiv \omega_{k}^{(1-\gamma)}, l=0, \ldots, k$. Then, for the generating function $\omega(z, \alpha)=(1-z)^{\alpha}$, by Eq. (8) we get

${ }_{0} D_{t}^{1-\gamma} u\left(x_{j}, t_{k}\right)=\tau^{\gamma-1} \sum_{l=0}^{k} \lambda_{l} u\left(x_{j}, t_{k-l}\right)+\mathcal{O}(\tau)$

By (7) and (10), and backward difference formula

$\left.\frac{\partial u}{\partial t}\right|_{j} ^{k}=\frac{u_{j}^{k}-u_{j}^{k-1}}{\tau}+\mathcal{O}(\tau)$

the implicit CFD method for problem (1)-(4) is given as follows

$\frac{u_{j}^{k}-u_{j}^{k-1}}{\tau}=\kappa_{\gamma} \frac{\tau^{\gamma-1}}{h^{2}} \sum_{l=0}^{k} \lambda_{l} \frac{\delta_{x}^{2}}{1+\frac{1}{12} \delta_{x}^{2}} u_{j}^{k-l}-\kappa \tau^{\gamma-1} \sum_{l=0}^{k} \lambda_{l} u_{j}^{k-l}+f_{j}^{k}, \quad j=1,2, \ldots, M-1, \quad k=1,2, \ldots, N$

where

$u_{j}^{0}=\varphi\left(x_{j}\right), \quad j=1,2, \ldots, M-1$,

and

$u_{0}^{k}=\phi\left(t_{k}\right), \quad u_{M}^{k}=\psi\left(t_{k}\right), \quad k=1,2, \ldots, N$.

By multiplying both sides of (12) by the operator $\tau\left(1+\frac{1}{12} \delta_{x}^{2}\right)$, after rearranging the terms and noting that $\lambda_{0}=1$, we get

$$
\begin{aligned}
& {\left[1+\mu_{2}+\left(\frac{1+\mu_{2}}{12}-\mu_{1}\right) \delta_{x}^{2}\right] u_{j}^{k}=\left(1+\frac{1}{12} \delta_{x}^{2}\right) u_{j}^{k-1}+\mu_{1} \sum_{l=1}^{k} \lambda_{l} \delta_{x}^{2} u_{j}^{k-l}-\mu_{2} \sum_{l=1}^{k} \lambda_{l}\left(1+\frac{1}{12} \delta_{x}^{2}\right) u_{j}^{k-l}} \\
& +\tau\left(1+\frac{1}{12} \delta_{x}^{2}\right) f_{j}^{k}, \quad 1 \leq j \leq M-1, \quad 1 \leq k \leq N,
\end{aligned}
$$

where $\mu_{1}=\kappa_{\gamma} \frac{\tau^{\gamma}}{h^{2}}$ and $\mu_{2}=\kappa \tau^{\gamma}$.

So, for $k=1$, we obtain

$$
\begin{aligned}
& \left(\frac{1+\mu_{2}}{12}-\mu_{1}\right) u_{j-1}^{1}+\left[\frac{5}{6}\left(1+\mu_{2}\right)+2 \mu_{1}\right] u_{j}^{1}+\left(\frac{1+\mu_{2}}{12}-\mu_{1}\right) u_{j+1}^{1} \\
= & {\left[\frac{1}{12}+\lambda_{1}\left(\mu_{1}-\frac{\mu_{2}}{12}\right)\right] u_{j-1}^{0}+\left[\frac{5}{6}-\lambda_{1}\left(2 \mu_{1}+\frac{5 \mu_{2}}{6}\right)\right] u_{j}^{0}+\left[\frac{1}{12}+\lambda_{1}\left(\mu_{1}-\frac{\mu_{2}}{12}\right)\right] u_{j+1}^{0} } \\
+ & \frac{\tau}{12}\left(f_{j-1}^{1}+10 f_{j}^{1}+f_{j+1}^{1}\right),
\end{aligned}
$$

and for $2 \leq k \leq N$,

$$
\begin{aligned}
& \left(\frac{1+\mu_{2}}{12}-\mu_{1}\right) u_{j-1}^{k}+\left[\frac{5}{6}\left(1+\mu_{2}\right)+2 \mu_{1}\right] u_{j}^{k}+\left(\frac{1+\mu_{2}}{12}-\mu_{1}\right) u_{j+1}^{k} \\
& =\left[\frac{1}{12}+\lambda_{1}\left(\mu_{1}-\frac{\mu_{2}}{12}\right)\right] u_{j-1}^{k-1}+\left[\frac{5}{6}-\lambda_{1}\left(2 \mu_{1}+\frac{5 \mu_{2}}{6}\right)\right] u_{j}^{k-1}+\left[\frac{1}{12}+\lambda_{1}\left(\mu_{1}-\frac{\mu_{2}}{12}\right)\right] u_{j+1}^{k-1} \\
& +\sum_{l=2}^{k} \lambda_{l}\left[\left(\mu_{1}-\frac{\mu_{2}}{12}\right) u_{j-1}^{k-l}-\left(2 \mu_{1}+\frac{5 \mu_{2}}{6}\right) u_{j}^{k-l}+\left(\mu_{1}-\frac{\mu_{2}}{12}\right) u_{j+1}^{k-l}\right]+\frac{\tau}{12}\left(f_{j-1}^{k}+10 f_{j}^{k}+f_{j+1}^{k}\right) .
\end{aligned}
$$


By using the "empty sum" convention, we can write (16) and (17) simply as

$$
\begin{aligned}
& \left(\frac{1+\mu_{2}}{12}-\mu_{1}\right) u_{j-1}^{k}+\left[\frac{5}{6}\left(1+\mu_{2}\right)+2 \mu_{1}\right] u_{j}^{k}+\left(\frac{1+\mu_{2}}{12}-\mu_{1}\right) u_{j+1}^{k} \\
& =\left[\frac{1}{12}+\lambda_{1}\left(\mu_{1}-\frac{\mu_{2}}{12}\right)\right] u_{j-1}^{k-1}+\left[\frac{5}{6}-\lambda_{1}\left(2 \mu_{1}+\frac{5 \mu_{2}}{6}\right)\right] u_{j}^{k-1}+\left[\frac{1}{12}+\lambda_{1}\left(\mu_{1}-\frac{\mu_{2}}{12}\right)\right] u_{j+1}^{k-1} \\
& +\sum_{l=0}^{k-2} \lambda_{k-l}\left[\left(\mu_{1}-\frac{\mu_{2}}{12}\right) u_{j-1}^{l}-\left(2 \mu_{1}+\frac{5 \mu_{2}}{6}\right) u_{j}^{l}+\left(\mu_{1}-\frac{\mu_{2}}{12}\right) u_{j+1}^{l}\right] \\
& +\frac{\tau}{12}\left(f_{j-1}^{k}+10 f_{j}^{k}+f_{j+1}^{k}\right), \quad 1 \leq j \leq M-1, \quad 1 \leq k \leq N .
\end{aligned}
$$

\section{Matrix form of the numerical scheme}

By multiplying both sides of (18) by a common factor 12, the matrix form of the scheme is given by

$$
\left\{\begin{array}{l}
A U^{1}=\widetilde{B}_{0} U^{0}+F^{1} \\
A U^{k}=\sum_{l=0}^{k-1} B_{l}^{k} U^{l}+F^{k}, \quad k=2,3, \ldots, N
\end{array}\right.
$$

where the tridiagonal matrices $A, \widetilde{B}_{0}$ and $B_{l}^{k}(0 \leq l \leq k-1)$ are given by

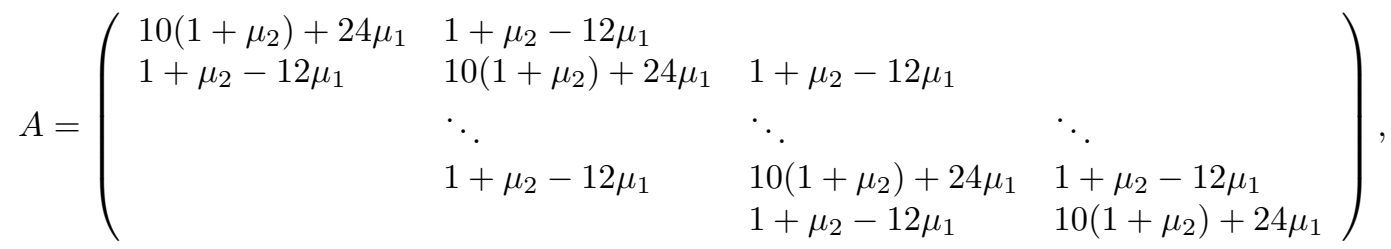

$$
\begin{aligned}
& \widetilde{B}_{0}=\left(\begin{array}{llll}
10-\lambda_{1}\left(24 \mu_{1}+10 \mu_{2}\right) & 1-\lambda_{1}\left(\mu_{2}-12 \mu_{1}\right) & & \\
1-\lambda_{1}\left(\mu_{2}-12 \mu_{1}\right) & 10-\lambda_{1}\left(24 \mu_{1}+10 \mu_{2}\right) & 1-\lambda_{1}\left(\mu_{2}-12 \mu_{1}\right) & \\
& \ddots & \ddots & \ddots \\
& 1-\lambda_{1}\left(\mu_{2}-12 \mu_{1}\right) & 10-\lambda_{1}\left(24 \mu_{1}+10 \mu_{2}\right) & 1-\lambda_{1}\left(\mu_{2}-12 \mu_{1}\right) \\
& 1-\lambda_{1}\left(\mu_{2}-12 \mu_{1}\right) & 10-\lambda_{1}\left(24 \mu_{1}+10 \mu_{2}\right)
\end{array}\right), \\
& B_{l}^{k}=\lambda_{k-l}\left(\begin{array}{llll}
-24 \mu_{1}-10 \mu_{2} & 12 \mu_{1}-\mu_{2} & & \\
12 \mu_{1}-\mu_{2} & -24 \mu_{1}-10 \mu_{2} & 12 \mu_{1}-\mu_{2} & \\
& \ddots & \ddots & \ddots \\
& 12 \mu_{1}-\mu_{2} & -24 \mu_{1}-10 \mu_{2} & 12 \mu_{1}-\mu_{2} \\
& & 12 \mu_{1}-\mu_{2} & -24 \mu_{1}-10 \mu_{2}
\end{array}\right), \quad l=0,1, \ldots, k-2
\end{aligned}
$$

and $B_{k-1}^{k}=\widetilde{B}_{0}, \quad k \geq 2$.

Furthermore, the column vectors $F^{1}$ and $F^{k}$ in (19) are as follows

$$
F^{1}=\left(\begin{array}{c}
{\left[1-\lambda_{1}\left(\mu_{2}-12 \mu_{1}\right)\right] u_{0}^{0}-\left(1+\mu_{2}-12 \mu_{1}\right) u_{0}^{1}+\tau\left(f_{0}^{1}+10 f_{1}^{1}+f_{2}^{1}\right)} \\
\tau\left(f_{1}^{1}+10 f_{2}^{1}+f_{3}^{1}\right) \\
\vdots \\
\tau\left(f_{M-3}^{1}+10 f_{M-2}^{1}+f_{M-1}^{1}\right) \\
{\left[1-\lambda_{1}\left(\mu_{2}-12 \mu_{1}\right)\right] u_{M}^{0}-\left(1+\mu_{2}-12 \mu_{1}\right) u_{M}^{1}+\tau\left(f_{M-2}^{1}+10 f_{M-1}^{1}+f_{M}^{1}\right)}
\end{array}\right),
$$


and

$$
F^{k}=\left(\begin{array}{c}
\sum_{l=0}^{k-2} \lambda_{k-l}\left(12 \mu_{1}-\mu_{2}\right) u_{0}^{l}+\left[1-\lambda_{1}\left(\mu_{2}-12 \mu_{1}\right)\right] u_{0}^{k-1}-\left(1+\mu_{2}-12 \mu_{1}\right) u_{0}^{k}+\tau\left(f_{0}^{k}+10 f_{1}^{k}+f_{2}^{k}\right) \\
\tau\left(f_{1}^{k}+10 f_{2}^{k}+f_{3}^{k}\right) \\
\vdots \\
\tau\left(f_{M-3}^{k}+10 f_{M-2}^{k}+f_{M-1}^{k}\right) \\
\sum_{l=0}^{k-2} \lambda_{k-l}\left(12 \mu_{1}-\mu_{2}\right) u_{M}^{l}+\left[1-\lambda_{1}\left(\mu_{2}-12 \mu_{1}\right)\right] u_{M}^{k-1}-\left(1+\mu_{2}-12 \mu_{1}\right) u_{M}^{k}+\tau\left(f_{M-2}^{k}+10 f_{M-1}^{k}+f_{M}^{k}\right)
\end{array}\right),
$$

for $k \geq 2$.

Theorem 3.1 The difference system (15) is solvable.

Proof. Because for any $\mu_{1}=\kappa_{\gamma} \frac{\tau^{\gamma}}{h^{2}}>0$ and $\mu_{2}=\kappa \tau^{\gamma}>0$, the coefficient matrix A in (19) is strictly diagonally dominant. Consequently the matrix A is invertible and the system (15) has a unique solution.

\section{Theoretical analysis of the CFD scheme}

\subsection{Stability}

We investigate the stability of the CFD scheme by the Fourier method. Let $\rho_{j}^{k}$ be the roundoff error in the mesh point $(j h, k \tau)$. Noticing $(15)$, the following roundoff error equations are obtained

$$
\begin{aligned}
& {\left[1+\mu_{2}+\left(\frac{1+\mu_{2}}{12}-\mu_{1}\right) \delta_{x}^{2}\right] \rho_{j}^{k}=\left(1+\frac{1}{12} \delta_{x}^{2}\right) \rho_{j}^{k-1}+\mu_{1} \sum_{l=1}^{k} \lambda_{l} \delta_{x}^{2} \rho_{j}^{k-l}-\mu_{2} \sum_{l=1}^{k} \lambda_{l}\left(1+\frac{1}{12} \delta_{x}^{2}\right) \rho_{j}^{k-l},} \\
& \rho_{0}^{k}=\rho_{M}^{k}=0, \quad 1 \leq k \leq N .
\end{aligned}
$$

We let

$\rho^{k}=\left(\rho_{1}^{k}, \rho_{2}^{k}, \ldots, \rho_{M-1}^{k}\right)^{T}$,

and introduce the following norm

$\left\|\rho^{k}\right\|_{l^{2}}=\left(\sum_{j=1}^{M-1} h\left|\rho_{j}^{k}\right|^{2}\right)^{1 / 2}$.

Now, suppose that the solutions of equations (20) and (21) have the following form

$\rho_{j}^{k}=G_{k} e^{i \sigma j h}, \quad j=1,2, \ldots, M-1, k=1,2, \ldots, N$,

where $\sigma=2 \pi n / L(n \in Z)$. Substituting the above expression into (20), we get

$$
\begin{aligned}
{\left[1+\mu_{2}+\left(-\frac{1+\mu_{2}}{3}+4 \mu_{1}\right) \sin ^{2}\left(\frac{\sigma h}{2}\right)\right] G_{k}=} & {\left[1-\lambda_{1} \mu_{2}+\left(-\frac{1-\lambda_{1} \mu_{2}}{3}-4 \lambda_{1} \mu_{1}\right) \sin ^{2}\left(\frac{\sigma h}{2}\right)\right] G_{k-1} } \\
& -\left[\mu_{2}+\left(-\frac{\mu_{2}}{3}+4 \mu_{1}\right) \sin ^{2}\left(\frac{\sigma h}{2}\right)\right] \sum_{l=0}^{k-2} \lambda_{k-l} G_{l}, \quad 1 \leq k \leq N .
\end{aligned}
$$

Consequently,

$$
\left\{\begin{array}{l}
G_{1}=\frac{1-\lambda_{1} \mu_{2}+\left(-\frac{1-\lambda_{1} \mu_{2}}{3}-4 \lambda_{1} \mu_{1}\right) \sin ^{2}\left(\frac{\sigma h}{2}\right)}{1+\mu_{2}+\left(-\frac{1+\mu_{2}}{3}+4 \mu_{1}\right) \sin ^{2}\left(\frac{\sigma h}{2}\right)} G_{0}, \\
G_{k}=\frac{1-\lambda_{1} \mu_{2}+\left(-\frac{1-\lambda_{1} \mu_{2}}{3}-4 \lambda_{1} \mu_{1}\right) \sin ^{2}\left(\frac{\sigma h}{2}\right)}{1+\mu_{2}+\left(-\frac{1+\mu_{2}}{3}+4 \mu_{1}\right) \sin ^{2}\left(\frac{\sigma h}{2}\right)} G_{k-1}-\frac{\mu_{2}+\left(-\frac{\mu_{2}}{3}+4 \mu_{1}\right) \sin ^{2}\left(\frac{\sigma h}{2}\right)}{1+\mu_{2}+\left(-\frac{1+\mu_{2}}{3}+4 \mu_{1}\right) \sin ^{2}\left(\frac{\sigma h}{2}\right)} \sum_{l=0}^{k-2} \lambda_{k-l} G_{l}, \quad 2 \leq k \leq N .
\end{array}\right.
$$


Lemma 4.1 The coefficients $\lambda_{l}(l=0,1, \ldots)$ satisfy [17]

(1) $\lambda_{0}=1, \quad \lambda_{1}=\gamma-1, \quad \lambda_{l}<0, l=1,2, \ldots$

(2) $\sum_{l=0}^{\infty} \lambda_{l}=0$, and for all $n \geq 1,-\sum_{l=1}^{n} \lambda_{l}<1$.

Thus we can proof following lemma

Lemma 4.2 Suppose that $G_{k}(1 \leq k \leq N)$ are defined by (26), then for $0<\gamma<1$, we have

$\left|G_{k}\right| \leq\left|G_{0}\right|, \quad k=1,2, \ldots, N$.

Proof. We prove the lemma by mathematical induction. For $\mathrm{k}=1$, from the first equation in (26) we have

$\left|G_{1}\right| \leq \frac{1-\lambda_{1} \mu_{2}+\left(-\frac{1-\lambda_{1} \mu_{2}}{3}-4 \lambda_{1} \mu_{1}\right) \sin ^{2}\left(\frac{\sigma h}{2}\right)}{1+\mu_{2}+\left(-\frac{1+\mu_{2}}{3}+4 \mu_{1}\right) \sin ^{2}\left(\frac{\sigma h}{2}\right)}\left|G_{0}\right|$.

From the lemma 1, we have $-1<\lambda_{1}<0$ and consequently $\left|G_{1}\right| \leq\left|G_{0}\right|$. Now suppose that $\left|G_{k}\right| \leq\left|G_{0}\right| ; 1 \leq k \leq n-1$. By this hypothesis and the second equation in (26), we get

$\left|G_{n}\right| \leq\left[\frac{1-\lambda_{1} \mu_{2}+\left(-\frac{1-\lambda_{1} \mu_{2}}{3}-4 \lambda_{1} \mu_{1}\right) \sin ^{2}\left(\frac{\sigma h}{2}\right)}{1+\mu_{2}+\left(-\frac{1+\mu_{2}}{3}+4 \mu_{1}\right) \sin ^{2}\left(\frac{\sigma h}{2}\right)}+\frac{\mu_{2}+\left(-\frac{\mu_{2}}{3}+4 \mu_{1}\right) \sin ^{2}\left(\frac{\sigma h}{2}\right)}{1+\mu_{2}+\left(-\frac{1+\mu_{2}}{3}+4 \mu_{1}\right) \sin ^{2}\left(\frac{\sigma h}{2}\right)} \sum_{l=0}^{n-2}\left|\lambda_{n-l}\right|\right]\left|G_{0}\right|$.

By the lemma 1, we can write

$\sum_{l=0}^{n-2}\left|\lambda_{n-l}\right|=\sum_{l=0}^{n-1}\left|\lambda_{n-l}\right|-\left|\lambda_{1}\right|<1+\lambda_{1}$.

Now, from (27) and (28) we obtain $\left|G_{n}\right| \leq\left|G_{0}\right|$. Hence the proof is completed.

Theorem 4.3 The implicit CFD scheme defined by (15) is unconditionally stable for $0<\gamma<1$.

Proof. From (23) and (24), and by applying Lemma 2, we have

$\left\|\rho^{k}\right\|_{l^{2}}^{2}=h \sum_{j=1}^{M-1}\left|G_{k} e^{i \sigma j h}\right|^{2}=h \sum_{j=1}^{M-1}\left|G_{k}\right|^{2} \leq h \sum_{j=1}^{M-1}\left|G_{0}\right|^{2}=h \sum_{j=1}^{M-1}\left|G_{0} e^{i \sigma j h}\right|^{2}=\left\|\rho^{0}\right\|_{l^{2}}^{2}, \quad k=1,2, \ldots, N$.

Hence the unconditionally stability of the scheme is proved.

By Eqs. (7), (10) and (11), the local truncation error of the scheme (15) is obtained as follows

$$
\begin{aligned}
R_{j}^{k}= & \frac{u\left(x_{j}, t_{k}\right)-u\left(x_{j}, t_{k-1}\right)}{\tau}-\kappa_{\gamma} \frac{\tau^{\gamma-1}}{h^{2}} \sum_{l=0}^{k} \lambda_{l} \frac{\delta_{x}^{2}}{1+\frac{1}{12} \delta_{x}^{2}} u\left(x_{j}, t_{k-l}\right)+\kappa \tau^{\gamma-1} \sum_{l=0}^{k} \lambda_{l} u\left(x_{j}, t_{k-l}\right)-f\left(x_{j}, t_{k}\right) \\
= & \frac{\partial}{\partial t} u\left(x_{j}, t_{k}\right)-\kappa_{\gamma} \tau^{\gamma-1} \sum_{l=0}^{k} \lambda_{l}\left(\frac{\partial^{2}}{\partial x^{2}} u\left(x_{j}, t_{k-l}\right)-\frac{h^{4}}{240} \frac{\partial^{6}}{\partial x^{6}} u\left(x_{j}, t_{k-l}\right)+\ldots\right) \\
& +\kappa \tau^{\gamma-1} \sum_{l=0}^{k} \lambda_{l} u\left(x_{j}, t_{k-l}\right)-f\left(x_{j}, t_{k}\right)+\mathcal{O}(\tau) \\
= & \frac{\partial}{\partial t} u\left(x_{j}, t_{k}\right)-{ }_{0} D_{t}^{1-\gamma}\left[\kappa_{\gamma} \frac{\partial^{2} u\left(x_{j}, t_{k}\right)}{\partial x^{2}}-\kappa u\left(x_{j}, t_{k}\right)\right]-f\left(x_{j}, t_{k}\right) \\
& +\kappa_{\gamma}\left(\frac{h^{4}}{240}\right){ }_{0} D_{t}^{1-\gamma} \frac{\partial^{6}}{\partial x^{6}} u\left(x_{j}, t_{k}\right)+\mathcal{O}(\tau)+\mathcal{O}\left(h^{6}\right) \\
= & \mathcal{O}\left(\tau+h^{4}\right) .
\end{aligned}
$$

Also, the consistency of the scheme is concluded through above relations. 


\section{Numerical experiments}

We applied the method presented in this paper and solved 1 example given in [2].

Example 1. Consider [2],

$$
\begin{aligned}
& \frac{\partial u(x, t)}{\partial t}={ }_{0} D_{t}^{1-\gamma}\left[\frac{\partial^{2} u(x, t)}{\partial x^{2}}-u(x, t)\right]+(1+\gamma) e^{x} t^{\gamma}, \quad 0<t \leq 1, \quad 0<x<1, \\
& u(x, 0)=0, \quad 0 \leq x \leq 1, \\
& u(0, t)=t^{1+\gamma}, \quad u(1, t)=e t^{1+\gamma}, \quad 0<t \leq T .
\end{aligned}
$$

The exact solution of the problem is $u(x, t)=e^{x} t^{1+\gamma}$.

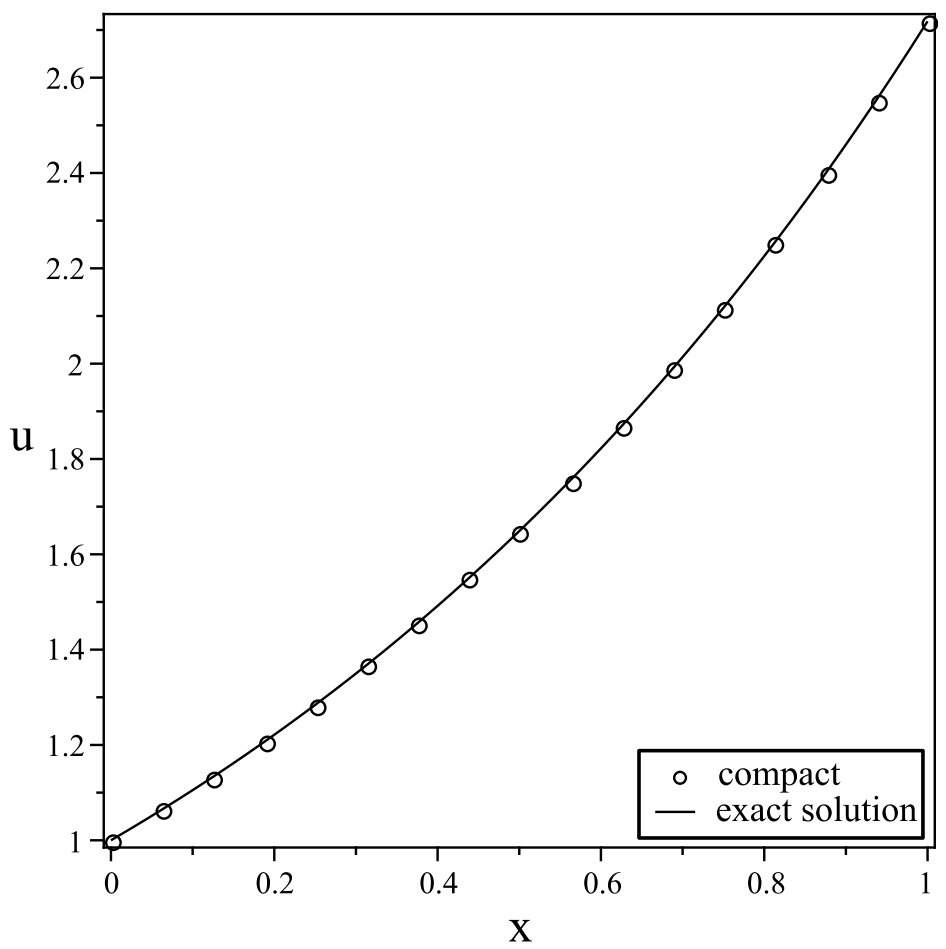

Figure 1: CFD solutions with $h=1 / 16$ and $\tau=1 / 8$ together with the exact solution at $T=1(\gamma=0.8)$.

We solved the problem (29)-(31) by the method presented in this paper. In Table 1, the maximum error for numerical solutions and the experimental convergence order for $\gamma=0.25$ and different values of $\tau$ and $h$, are shown. The followings are used for the maximum error and the experimental convergence order $(C-O r d e r)$.

$\|e\|_{l \infty}=\max _{1 \leq j \leq M-1}\left\{\left|u\left(x_{j}, 1\right)-u_{j}^{N}\right|\right\}$,

and

$C-$ Order $=\log _{2}\left(\|e(16 \tau, 2 h)\|_{l^{\infty}} /\|e(\tau, h)\|_{l^{\infty}}\right)$,

in which $\|e(\tau, h)\|_{l_{\infty}}$ means the error $\|e\|_{l_{\infty}}$ computed with mesh sizes $\tau$ and $h$. The comparison between the exact solution and the numerical solutions of CFD method for $h=1 / 16 ; \tau=1 / 8$ and $\gamma=0.8$ at $T=1$ is shown in Fig. 1 .

\section{Conclusion}

In this paper, an implicit CFD scheme for FR-subDE is presented. By the method, the computation of FR-subDE is reduced to some linear systems with a tridiagonal coefficient matrix. So, the systems are easy to solve. The 
Table 1: Maximum error and experimental convergence order for our CFD scheme $(\gamma=0.25)$.

\begin{tabular}{ccc}
\hline Mesh sizes & $\|e\|_{l^{\infty}}$ & $C-$ Order \\
\hline $\mathrm{h}=\tau=1 / 4$ & $1.47615 \times 10^{-2}$ & - \\
$\mathrm{h}=1 / 8, \tau=1 / 64$ & $1.24074 \times 10^{-3}$ & 3.57 \\
$\mathrm{~h}=1 / 16, \tau=1 / 1024$ & $8.78350 \times 10^{-5}$ & 3.82 \\
\hline
\end{tabular}

method is unconditionally stable for $0<\gamma<1$ and it has accuracy of four in the spatial grid size and one in the time step. Numerical results are in the agreement with the our theoretical findings.

\section{References}

[1] W.F. Ames, Numerical Methods for Partial Differential Equations, Academic Press, New York, 1977.

[2] C. M. Chen, F. Liu and K. Burrage, Finite difference methods and a fourier analysis for the fractional reactionsubdiffusion equation, Appl. Math. Comput. 198 (2008) 754-769.

[3] M. Cui, Compact finite difference method for the fractional diffusion equation, J. Comp. Phys. 228 (2009) $7792-7804$.

[4] M.R. Cui, Fourth-order Scheme for the one dimensional Sine-Gordon equation, Numer. Meth. Partial Diff. Eq. 25 (2009) 685-711.

[5] B.I. Henry, S.L. Wearne, Fractional reaction-diffusion, Physica. A 276 (2000) 448-455.

[6] T.A.M. Langlands, B.I. Henry, The accuracy and stability of an implicit solution method for the fractional diffusion equation, J. Comp. Phys. 205 (2005) 719-736.

[7] W. Liao, J. Zhu, A.Q.M. Khaliq, An efficient high order algorithm for solving systems of reaction-diffusion equations, Numer. Meth. Partial Diff. Eq. 18 (2002) 340-354.

[8] F. Liu, V. Anh, I. Turner, Numerical solution of the space fractional Fokker-Planck equation, J. Comp. Appl. Math. 166 (2004) 209-219.

[9] F. Liu, V. Anh, I. Turner, P. Zhuang, Numerical simulation for solute transport in fractal porous media, ANZIAM J. Comp. 45 (E) (2004) 461-473.

[10] Q. Liu, F. Liu, I. Turner, V. Anh, Approximation of the Levy-Feller advection dispersion process by random walk and finite difference method, J. Comp. Phys. 222 (2007) 57-70.

[11] C.H. Lubich, Discretized fractional calculus, SIAM J. Math. Anal. 17 (3) (1986) 704-719.

[12] M. Meerschaert, C. Tadjeran, Finite difference approximations for fractional advection dispersion equation, J. Appl. Math. Comput. 172 (2004) 65-77.

[13] I. Podlubny, Fractional Differential Equations, Academic Press, San Diego, 1999.

[14] J.P. Roop, Computational aspects of FEM approximation of fractional advection dispersion equation on bounded domains in $R^{2}$, J. Comp. Appl. Math. 193 (1) (2006) 243-268.

[15] K. Seki, M. Wojcik, M. Tachiya, Fractional reaction-diffusion equation, J. Chem. Phys., 119 (2003) $2165-2174$.

[16] S.B. Yuste, L. Acedo, An explicit finite difference method and a new Von Neumann-type stability analysis for fractional diffusion equations, SIAM J. Numer. Anal. 42 (5) (2005) 1862-1874.

[17] P. Zhuang, F. Liu, Implicit difference approximations for the time fractional diffusion equation, J. Appl. Math. Comput. 22 (3) (2006) 87-99. 